Check for updates

Cite this as: BMJ 2021;372:n860 http://dx.doi.org/10.1136/bmj.n860 Published: 31 March 2021

\section{Update to living WHO guideline on drugs for covid-19}

This living guideline by Lamontagne and colleagues (BMJ 2020;370:m3379) has been updated. For the latest update, visit doi:. The latest version of this WHO living guidance provides a recommendation against ivermectin in patients with covid-19 regardless of disease severity, except in the context of a clinical trial. 